\title{
Framework for Spectrum Authorization Elements and its Application to 5G Micro-Operators
}

\author{
K. B. Shashika Manosha, Marja Matinmikko-Blue and Matti Latva-aho \\ Centre for Wireless Communications (CWC) \\ University of Oulu \\ Oulu, Finland \\ manosha.kapuruhamybadalge@oulu.fi; marja.matinmikko@oulu.fi; matti.latva-aho@oulu.fi
}

\begin{abstract}
The fifth generation (5G) networks are expected to deliver high quality services to human and machine type users in specific locations. The recently proposed micro-operator concept aims at efficient and reliable local service delivery to complement traditional mobile network operators (MNOs) whose main objective has been to provide country-wide coverage. The uptake of 5G micro-operators requires local spectrum availability. In this paper, we propose a general framework for spectrum authorization and apply it in the recent micro-operator concept to develop local spectrum micro licensing model. To do this, we first identify the elements of a spectrum authorization model, and derive their interrelations to form a general framework for spectrum authorization. Then, we analyze the elements of the state-of-the-art spectrum authorization models including case examples of exclusive licensing, three-tier model for providing citizens broadband radio services (CBRS), and license-exempt model. Using the framework and the state-of-the-art models, we then propose and characterize the elements of the new micro licensing model to establish local 5G networks. Furthermore, our comprehensive investigation on the new micro licensing model supports to establish the micro-operator concept as a candidate solution for delivering localized 5G services. The proposed framework and its application to 5G micro-operators help to promote new market entry, encourage competition and innovation, and incentivize investment by facilitating the regulator to efficiently allocate $5 \mathrm{G}$ bands to the stakeholders.
\end{abstract}

Keywords - Spectrum authorization model; licensing elements; CBRS; SAS; LSA; 5G; mobile network operator; small cell; spectrum sharing

\section{INTRODUCTION}

The future fifth generation $(5 \mathrm{G})$ networks will play a major role in service delivery in the areas like automotive, smart society, smart grids, health, logistics, etc [1]. These networks will address a large number of diverse use cases, which have been categorized into three broad types, based on their requirements: massive machine type communication, critical machine type communication, and enhanced mobile broadband [2]. Most of the specific scenarios within these use cases, such as factory cell automation, outdoor events, remote surgery and examination, shopping malls, virtual and augmented reality, need location specific services. Hence, to provide $5 \mathrm{G}$ services that require high data rates with low latency, it is essential to

Authors would like to acknowledge the funding from Tekes - the Finnish Funding Agency for Innovation in $\mathrm{uO5G}$ project. have locally operated dense deployments of 5G networks [3].

At present, the mobile broadband connectivity for end users is provided mostly by MNOs with cellular networks and fixed network operators through wireless local area networks (WLAN). The main target of the MNOs is to ensure countrywide coverage, thus offering services for a mass quantity of subscribers over large areas. On the other hand, most of the mobile traffic originates from indoors [3], which is carried by WLAN to a large extent. Furthermore, handling the mobile traffic generated by heavy data consuming applications in indoor areas has been challenging to the MNOs to serve from outdoor cellular networks, because the network coverage severely degrades inside the buildings due to building penetration losses. Thus, it is an open question how to provide high quality localized services planned to be offered via $5 \mathrm{G}$ networks to the customers with the MNOs existing infrastructure, especially inside large buildings like shopping malls, factory premises, etc.

To address the above, the emergence of a new role to the mobile market has led to the development of the microoperator concept [4]-[6]. A micro-operator is a local and possibly venue specific service provider that deploys and operates a local small cell network and offers reliable high quality $5 \mathrm{G}$ related local services. It is a role that can be taken by different stakeholders, such as facility owners, with different levels of infrastructure ownership to serve different customer sets. For example, the micro-operator could operate a neutral-host network inside a mall or an arena to serve MNOs' customers [6]. On the other hand, it could serve a closed customer set by running a private network (e.g., factory [6]), or a mix of MNO customers and its own customers.

Reliable delivery of high quality $5 \mathrm{G}$ services and new network deployment models are highly dependent on the spectrum authorization decisions for $5 \mathrm{G}$ bands taken by the regulators, which is an open topic with emerging options for more dynamic approaches [7]. While MNOs continue to require guaranteed access to spectrum, the emergence of local micro-operators will also need guaranteed local spectrum availability to offer high quality localized $5 \mathrm{G}$ services. Hence, an important challenge in micro-operator concept is how to gain spectrum access rights. At present, the existing spectrum authorization models for mobile broadband include exclusive licensing (see, e.g. [8]) and license-exempt spectrum access 
(see e.g. [9]). They are expanded, more flexibly, in the US citizens broadband service radio (CBRS) [10]-[11] and European Licensed Shared Access (LSA) approaches [12]. License-exempt spectrum access [9] alone is not adequate for the micro-operator concept as it cannot provide guaranteed spectrum access, while the facility owner has some level of control on who can transmit within its premises [3]. Exclusive licensing for mobile communications typically provides guaranteed spectrum access for a smaller group of operators for a very long period of time (usually $15-20$ years), and hence, does not directly accommodate a large number of local microoperators. There are no discussions about applying European LSA model [12] to this yet. Thus, there are no specific authorization models that would introduce additional licensed users to bands already licensed for other purposes. The closest fit to micro-operators is the US three-tier model for CBRS [10]-[11] that introduces a priority access license (PAL) layer with local licenses and a general authorized access (GAA) layer for license-exempt use while protecting incumbent users through a spectrum access system (SAS) for interference coordination. The key difference between LSA and CBRS is the flexibility of obtaining spectrum access rights provided in CBRS; a user can decide whether to be a PAL or GAA user depending on the level of interference protection he needs and the amount of money he is willing to spend. Similarly, entrant micro-operators could apply for local PAL or operate in the GAA layer; but being GAA users the micro-operators may suffer from harmful interference. Therefore, there is a need for a new spectrum authorization model to allow for new entrant micro-operators in $5 \mathrm{G}$.

The development of new local spectrum authorization models requires a framework to define the elements of it. In regulation, for example in Europe, the authorization aspects are discussed in [13] and are being revised in [14]. However, the existing research literature lacks such a general spectrum authorization framework and the details are scattered. Closer to a framework is a process model for recent spectrum sharing models adopted in regulation [16] that defines the common steps including regulatory preparations, spectrum access rights, deployment, operations, and release. But, this process model does not fully present the details of spectrum authorization model elements. In our recent paper [15], we have studied 5G regulations and identified the preliminary elements of micro licensing model. We have provided a brief explanation of the elements without highlighting their relations or focusing on the connections it has to the existing authorization models.

In this paper, we propose a novel, generalized spectrum authorization framework to characterize existing and develop new authorization models and apply it to $5 \mathrm{G}$ spectrum regulation. To do this, we first identify the elements of spectrum authorization that are present in existing authorization models. Then, we develop a framework for spectrum authorization by identifying the relations between the elements. Here, we have observed that there are few elements (main elements) which have strong relations with rest of the elements. Building on top of [15], in this paper we review the state-ofthe-art spectrum authorization models including case examples of CBRS [10], [11], exclusive licensing [8], and licenseexempt spectrum access [9] using the proposed framework.
Finally, based on the developed framework, we propose a local spectrum authorization model for the micro-operators, i.e., micro licensing that was originally outlined in [5] and [15]. As the micro-operators' primary goal is to provide $5 \mathrm{G}$ services locally, the developed micro licensing model and guidelines are useful for developing generalized spectrum authorization models for upcoming $5 \mathrm{G}$ bands. Hence, our proposed framework is an ideal tool for a regulator to use in the future for efficiently allocating the spectrum among the stakeholders.

The rest of this paper is organized as follows. Section II defines the elements and framework for spectrum authorization. Section III provides a state of the art review of existing spectrum authorization models (CBRS, exclusive licensing, and license-except access) using the framework. Section IV introduces the key elements of the new micro licensing model followed by conclusions in Section V.

\section{DEFINING ELEMENTS AND FRAMEWORK FOR SPECTRUM AUTHORIZATION}

Identifying the elements of a spectrum authorization model is of paramount importance as it helps to define a precise framework for spectrum authorization. In this section, first, all the identified elements of a spectrum authorization model are discussed in detail; then a framework has been defined for it. The proposed framework is formed by understanding how strongly these elements are related with each other.

\section{A. Identification of elements of spectrum authorization model}

The elements of a spectrum authorization model include purpose of use, eligible licensee, license awarding procedure, technical conditions, license area, obligations, transferability of rights, and license duration, which are described in the following and illustrated in Fig. 1.

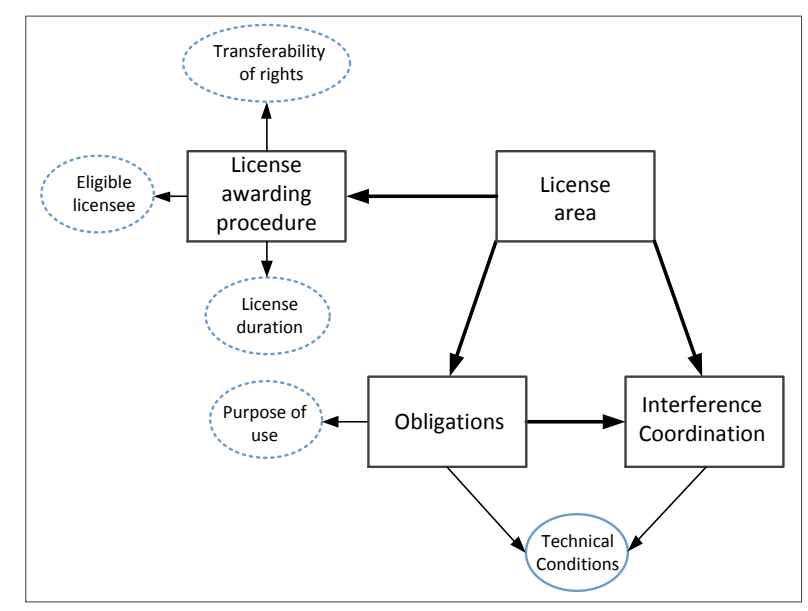

Fig. 1. Relation elements of a spectrum authorization framework.

Purpose of use defines the type of use allowed in the band. A regulator allocates spectrum bands for the provisioning of specific radio services. The purpose of use of the band ensures that the prospective licensees will operate according to the objectives of the regulator. The purpose of use can be defined by looking in different perspectives such as the deployment model, the varieties of users and use cases, and the business 
models. Hence, for example, the regulator may mandate that the prospective licensee can use the bands for mobile/fixed communication networks (MFCN) and it cannot be used for aeronautical mobile services. Precisely defining the purpose of use is significant, as this element has strong relations with the other important elements of a spectrum authorization model.

Eligible licensee defines who are eligible to apply and obtain a spectrum license in the band that the regulator is planning to authorize. This element acts as an initial filter to select the best set of candidates to participate in the process. In general, the regulator has the sole authority to define the criteria for selecting the eligible licensees, but should be done in a fair and transparent way to promote competition and innovation. Hence, the regulator could define criteria to select the eligible users by analyzing the level of public interest, investment in systems and services by entrants, spurring of investments, potential form of harmful interference towards other users, and national security related aspects. Note that being eligible does not guarantee a granted spectrum block.

License awarding procedure defines the process how the spectrum access rights are granted among the eligible licensees who are requesting for such rights. An efficient license awarding procedure needs to consider fairness and transparency to provide equal economic choices for the prospective eligible licensees. While beauty contest was often used in the past for license awarding in mobile communication, auction mechanisms have recently been used as the main license awarding procedure, see [17] and [18] for details. Auction design defines a competitive bidding procedure that can handle several applicants to bid for the same spectrum blocks. In addition to defining the mechanism, the detailed items to be defined in a license awarding procedure include band plan, size of the channels, and spectrum aggregation limits (the maximum number of spectrum blocks that can be acquired by a prospective licensee). Specifically, while determining the above items, the regulator may consider how an appropriate balance between permitting more entrants to the market and deciding the size of a block that suits for high data rate technologies can be obtained. Furthermore, having consistent with a harmonized channelization scheme, it provides additional benefits like low equipment complexity and global roaming facilities. Finally, the regulator should consider including a feedback collection approach in the development of license awarding.

License area is a geographical region defined by the regulator, within that a prospective licensee provides wireless communication services to its customers using the awarded band. Typically, country-wide license areas have been defined for mobile communications in existing mobile bands below 3 $\mathrm{GHz}$, but they face challenges in the new $5 \mathrm{G}$ bands in higher carrier frequencies. While defining the license area, the regulator can consider the following aspects: population density based geographic sizes, alignment with the natural features (rivers, mountains) and political subdivisions (city lines), demand focused divisions, availability of national data, and complexity of data management in the spectrum controllers. Typically, a licensee is obliged to provide some level of coverage over the license area. In the case where it is not meaningful to provide coverage for the entire license area, defining a service area is useful. That is, a geographical area within the license area of a licensee, and a reliable service can be guaranteed to a customer located inside the service area.

Interference coordination includes the criteria and mechanisms to define and guarantee the levels of protection required for service quality. In general, there can be spectrum licensees with equal and different priority levels in a particular spectrum authorization model. Hence, the interference coordination element facilitates coordinating the interference among the co-channel and adjacent channel users with different priorities, for efficient spectrum utilization. To do this, usually, methods (or tools) such as introducing exclusion and protection zones around the licensees, power control and beamforming techniques, and efficient frequency planning are needed.

Technical conditions include a set of least restrictive technical conditions and parameters to be followed by a license holder. The set of most critical parameters include, the block edge mask (BEM), interference protection margins, duplex spacing, maximum mean radiated power in the downlink and uplink, etc. Carefully defining the technical conditions of a spectrum authorization model is important, as it helps to provide a reliable service to the customers of a licensee and efficient utilization of the spectrum. Furthermore, the parameters defined in this element also affects the smooth operations conducted in the adjacent bands.

Obligations ensure that the objectives of the regulator are met by enforcing the licensees to follow a predefined set of operational constraints. For example, obligations are imposed to provide better service coverage, i.e., by mandating to cover a certain percentage of the population of the geographical area. Restricting to deploy a network where it can be a threat to the national security or observing strict transmission conditions near international borders and monitoring them carefully is another strict obligation. Moreover, operating on or below the imposed thresholds (like BEMs) also plays a major role under this element. However, it is worth noting that the obligations in a spectrum authorization model are defined on case by case, i.e., it depends on the band the regulator is going to authorize.

Transferability of rights refers to change in ownership of the rights of spectrum use. A regulator often facilitates an original spectrum licensee to transfer the rights of using the spectrum to a third party. However, this is often requested to be done under strict scrutiny of the regulator. Note that transferring spectrum usage rights in this way avoids the original spectrum licensee to use the spectrum in a communication network. On the other hand, the right of using the spectrum can be granted to a third party temporarily, when the original spectrum licensee is not actually using the acquired channel in certain parts of its license area. For example, if the service area of an original spectrum licensee is less than the license area, then the original licensee may transfer the spectrum access rights to a third party. However, transferring the rights in this way is possible based on the mutual agreements between the original licensee and the third party user. Spectrum exchanging can also be considered as a derived version of transferring the rights. Here, the spectrum is exchanged among the licensees according to their mutual interest under the supervision of the regulator. From the 
regulators point, it has to carefully re-evaluate the technical conditions (such as the ability to partition the spectrum, aggregation limits, etc.), legal directives, and other logistics to facilitate transferring the spectrum usage rights.

License duration defines the time period that a spectrum licensee can use the awarded band to provide wireless communication services. Traditionally, the license durations in mobile communications granted for MNOs have been long in the order of 10-20 years. It is clear that allowing a licensee to use the spectrum for a longer period of time will create a certain type of monopoly in market, and also it will discourage the new players to enter the market. Such facts may limit the investments and innovations in technology and services. On the other hand, shorter license durations must guarantee the return of investment in the business. Thus, there is a strong trade-off between the license duration and the investment. As a result, at present, new rules and policies are evolving to have both long and short term durations.

\section{B. Building a framework for analysis}

In this subsection, to build a framework for spectrum authorization, we first identified the main elements of the spectrum authorization model. Then, how these main elements are related with the other elements of the authorization model (see Fig. 1) are discussed. By observing how many strong relations exist for each of the above mentioned elements, we have identified that license awarding procedure, license area, obligations, and interference coordination, as the main elements required for a spectrum authorization model.

Relation between license awarding procedure and other elements: We have identified that the license awarding procedure has strong relations with the elements of eligible licensee, license duration, license area, and transferability of rights. Eligible licensee defines who are the most appropriate candidates to be in the license awarding procedure. While designing the license awarding procedure, the regulator considers the license duration of the awarded licenses and for which license areas the licenses should be awarded. For example, based on the spectrum demands in various geographical locations and population densities, the license awarding procedure design can be modified such that it leads to a high competition and efficient utilization of the spectrum. Furthermore, since the design depends on the band in question, it is essential that the period of the license duration satisfies the objectives of the license awarding procedure design. Thus, license duration and the license area are strongly connected with the license awarding procedure. Referring to the transferability of rights element, a licensee can transfer its usage rights to a third party only within its license area for a period less than or equal to the license duration. Hence, while designing the license awarding procedure the regulator has to think the conditions required for transferability of rights.

Relation between interference coordination and other elements: We have observed that license area and technical condition elements have strong relationships with the interference coordination, compared to the other elements in the spectrum authorization models. To efficiently manage the interference among the licensees, it is essential to carefully define the parameters that affect the co-channel and adjacent channel interference. For example, the BEM, maximum transmit power, and tolerable interference threshold levels etc., play a major role in deciding how much interference (either cochannel or adjacent channel) can be generated by the licensee's transmitter towards other licensees. Thus, there is a strong relationship between the interference coordination and technical condition elements. The notions of service and protection area, which are derived from the license area element, help the regulator to define necessary rules and conditions required for interference coordination. As an instance, the regulator may define the technical conditions such that the aggregate interference level within a service (or protection) area of a particular licensee is lesser than some predefined threshold. Given such conditions, the regulator can assess how many co-channel and adjacent channel users can efficiently operate in a certain geographical region without generating harmful interference to each other.

Relation between obligations and other elements: The licensee is obliged to follow the rules and conditions imposed by the regulator via the license area, technical conditions, interference coordination, and purpose of use elements. Specifically, the regulator awards the spectrum license to a prospective licensee for a clearly defined purpose (e.g., only for land mobile communication) in a predefined geographical area. Thus, it is clear that the license area and purpose of use elements have relations with obligations element. The licensee also has to adhere to the technical conditions imposed by the regulator. For example, it is necessary for a licensee to control its transmit power levels to satisfy the BEM and interference threshold levels, and follow other interference coordinating rules requested by the regulator to reduce interference (e.g., interference reporting, measuring, etc.).

Relation between license area and other elements: The license area has strong relations with obligations, interference coordination, and the license awarding procedure which were elaborated in the above discussions on the relations.

\section{ANALYSIS OF SPECTRUM AUTHORIZATION ELEMENTS IN STATE-OF-THE-ART MODELS}

In Section II we have identified and described the elements of a spectrum authorization model, and discussed how those elements are related to each other. In this section, the elements of state-of-the-art spectrum authorization models in the literature have been analyzed. Specifically, we focus on the case examples of CBRS in $3.5 \mathrm{GHz}$ in the US [10]-[11], the exclusive licensing with the example case of recent $700 \mathrm{MHz}$ band auction in Finland [8], and license-exempt spectrum access in $5 \mathrm{GHz}$ band in the UK [9]. It is worth noting that the above mentioned models are identified as the possible 5G spectrum authorization models by the regulators in different countries.

\section{A. CBRS in $3.5 \mathrm{GHz}$ in the US}

In CBRS [10]-[11], the purpose of use is to utilize the issued spectrum licenses to improve the broadband capacity. Primarily it allows provisioning of mobile services (except aeronautical mobile services). According to the FCC, the 
possible use cases for providing mobile services could be: 1) the MNOs can deploy small cells where they need additional capacity, 2) utilities, manufacturers and other large industries can construct private wireless broadband networks to automate processes, 3) can work as wireless backhaul solutions, etc.

The FCC has a broad authority while prescribing the features of eligible licensees, which have to be checked while allowing a prospective licensee to participate in the license awarding procedure. For example, the features like citizenship of the prospective licensee, its character (the role in the market), financial status, technical and other qualifications, ownership status of the business (whether it is foreign or local), and threats on national security are thoroughly considered.

The license awarding procedure in CBRS is for assigning PALs to the applicants; it is an auction conducted with respect to each census tract that presents the license area. The FCC intends to auction $100 \mathrm{MHz}$ from $3.5 \mathrm{GHz}$ band. This band is divided into 10 blocks each with a block size of $10 \mathrm{MHz}$, and only a maximum number of 7 channels will be auctioned in each census tract. The auction is conducted in the areas where there are more than one user is willing to bid. Hence, there will not be any auction in the areas where there are no enough competitors, and instead the whole band will be made available for GAA users. In that case, a PAL user who would like to obtain a license has to become a GAA user. While designing the license awarding procedure to maintain fairness, the band is divided among the two types of users (reserve a minimum of the band for GAA users in any given census tract).

The license area in CBRS is a census tract. This selection facilitates the licensees to compete for licenses only in the areas where they wish to provide services. Furthermore, from the spectrum controller's point of view (i.e., an SAS), having census tract based licensing eases the inclusion of census tract specific geographic and demographic data into an SAS. Critics for using census tract as the license area in CBRS is presented in [19].

Interference coordination in CBRS is an essential feature since there are three tiers of spectrum users (i.e., incumbent, PAL, and GAA users). The SAS has to coordinate the interference generated by the lower tier users towards the upper tier users, and the interference generated among the PAL users and among the GAA users. A tier-1 user (i.e., incumbent) is protected by introducing a protection zone around that incumbent's operational area. The simplest methodology that SAS uses to avoid interference towards the tier-1 users from the users in other tiers is to allocate orthogonal channels to those users who are within or near the protection area of that incumbent. For example, if a PAL or a GAA user is located within or very near to the protection area of an incumbent, the SAS make sure that it does not allocate the same channel (that is used by the incumbent) to any PAL or GAA user. To manage the interference towards the PAL users, the SAS introduces a default protection contour [11] $(-96 \mathrm{dBm} / 10 \mathrm{MHz})$ around the PAL user base station. Then, while allocating the channels to other PAL and GAA users (CBRS users), the SAS verifies that the aggregate co-channel interference power within the area covered by the default protect contour is less than $-96 \mathrm{dBm} / 10 \mathrm{MHz}$.
Technical conditions for CBRS are closely related to the interference coordination element. The specific values are presented in [10] and [11], omitted here due to space limitation.

Obligations in CBRS can be categorized as the commitments that the CBRS users are compelled to do with the SAS in different phases. For an instance, while registering the CBRS users in the SAS, they have to provide accurate information (location, technical parameters, licensing status, etc.). In the operational phase, the users have to report any location changes of their base stations, received signal strength values, and other performance measurements as requested by the SAS. Furthermore, the CBRS users are bound to use only the channels assigned by the SAS to them, and they have to report if the granted channel is not in use. In order to protect incumbents, these users have to release the channels as instructed by the SAS within the allowed time period.

Transferability of rights in CBRS model is allowed only for PAL users, as the GAA users do not own any rights of use. The PAL users can transfer their spectrum usage rights to another PAL or GAA user. However, before transferring the rights, it is essential to know whether that PAL user is actually using the channel or not. To do this, the FCC has proposed a criterion in which a PAL user is identified as "using the spectrum", if the received signal strength beyond the PAL protection area [11] is less than some threshold. In case if the PAL user has not defined a PAL protection area, then the SAS propose a default protection contour [10],[11] which may be larger than the PAL protection area. The SAS identifies the PAL user is not active if the received signal strength of the PAL user beyond the default protection area is less than some threshold. Thus, the PAL user can transfer its right to another user if the aforementioned criteria are satisfied.

License duration is currently defined to be a three-year license term for PAL users [11], but the discussions are still ongoing. The granted time window is non-renewable, except during the first application window. Hence, in the first application of a PAL, it is allowed to apply for two consecutive three-year terms. At the termination of the PAL rights, such PAL users are eligible to operate as GAA users in the same licensing area. For new PAL users, there will be interim filling windows conducted periodically. In the census tracts where there are unassigned PAL channels, interim application windows will be opened, before the expiration of an ongoing three-year PAL term.

\section{B. Exclusive licensing in $700 \mathrm{MHz}$ in Finland}

In Finland, the regulatory bodies involved in the issuance of spectrum licenses for mobile communications is the Ministry of Transport and Communications Finland (MINTC) that authorizes Finnish Communications Regulatory Authority (FICORA) to conduct the detailed process. Recently, MINTC awarded exclusive licenses in the $700 \mathrm{MHz}$ band to enhance mobile broadband capacity [8] through an auction conducted by FICORA. Next, we briefly describe the elements of this example of exclusive licensing of $700 \mathrm{MHz}$ band in Finland.

The purpose of use of issuing spectrum licenses in this band is to encourage more use of electronic communications services and to secure efficient usage of frequency spectrum. 
Furthermore, the regulator expects to improve nationwide availability, quality and capacity of wireless broadband connections in Finland. Any enterprise or organization can apply for a license, given that they have signed up before the given deadline. Thus, eligible licensee is any company or entity that fulfills the set of conditions for this band.

The license awarding procedure is an auction where a total of six $2 \times 5 \mathrm{MHz}$ frequency pairs was auctioned in the 703-733 $\mathrm{MHz}$ and $758-788 \mathrm{MHz}$ bands. To limit the number of channels per prospective licensee, a maximum of two $2 \times 5 \mathrm{MHz}$ frequency pairs was allowed to any individual enterprise or an organization. The highest valid bid placed for a specific band (or bands) was selected as the winner at the conclusion of the auction. The license area is nationwide except in the Alland islands and the license duration is a period of 17 years.

Next, we summarize the obligations related to this example exclusive licensing model. The operations can be started on the date specified by the FICORA (license beginning period), and all the license holders are required to start operations within two years from the date of license beginning period. The license holders are bound to remove any disruptions caused to other regulation-compliant radio communications (i.e., TV broadcasters and their reception) by their operations in this band. Furthermore, a license holder is obliged to cover $99 \%$ of the population of mainland Finland, within three years of the start of the license period, and $35 \%$ of this coverage requirement must be satisfied by the licensee's own network. Specifically, the licensee is required to provide coverage in all the main roads, secondary roads, regional roads, connecting roads in mainland Finland, and the entire rail network. Moreover, the licensee must be able to guarantee a reasonable indoor coverage within the coverage area, without any additional charges from its subscribers. In addition, the licensee is bounded to demonstrate such coverage availability when it is needed. However, such stringent coverage requirement is not needed near to the international borders like with Russia. Furthermore, a licensee may achieve the coverage requirement targets using its existing networks which are operating in 2.6 $\mathrm{GHz}, 1800 \mathrm{GHz}$, and $800 \mathrm{MHz}$ bands. Finally, the licensee must make the data (such as network coverage information) sufficiently available to it subscribers or public.

For efficient spectrum utilization, transferability of rights is facilitated to allow a licensee to transfer its licenses or parts of them under the approval of the regulator. On the other hand, a license holder may also lease the rights to use the spectrum to another licensee, given the approval of the regulator.

\section{License-Exempt Spectrum Access in $5 \mathrm{GHz}$ in $U K$}

In the case of license-exempt access, the same spectrum authorization elements are involved but there are no specific issued licenses. Thus, the meanings of the elements are different from the previous two cases. Here we use the UK license-exempt access in the $5 \mathrm{GHz}$ band as an example [9]. Purpose of use accommodates a range of different systems that operate in short range and coexist in the license-exempt band. Eligible licensee refers to who deploys equipment that are certified to fulfil the criteria for operations in the band which in general is not restricting the potential stakeholders. The license awarding procedure as such is absent but goes through the process of standardization to develop systems that fulfil regulatory criteria to be deployed in the license-exempt band. License area is related to the operational area restricted by the maximum transmission power levels.

Interference coordination mechanisms include the protection of incumbents in the band as well as sharing and coexistence mechanisms between the users of the licenseexempt band. Specifically, dynamic frequency selection is required to change the operational frequency when certain radio systems that use the same frequency are near and transmission power control is also requested in some cases. Technical conditions are defined similarly as in other authorization models including the least restrictive technical conditions and parameters such as the maximum transmission powers and out of band emission levels.

Obligations are related to obeying the technical conditions and interference coordination mechanisms that are required for the coexistence and sharing (e.g., duty cycles). Transferability of rights in this case is not meaningful as there are no such individually assigned rights, but all can access when the rules are fulfilled. License duration can be interpreted as the time duration when the use of the band is possible. The specialty for license-exempt access is that it is considered to be irreversible as removing of the devices from the market will be challenging. It is difficult to change the access rights in the band due to a potentially large number of deployed systems by lots of stakeholders and all equipment would need to be withdrawn from the market, which is cumbersome.

\section{DEFINING SPECTRUM AUthORIZATION ELEMENTS OF MICRO-OPERATOR SPECTRUM LICENSING}

Using the elements that are involved in all spectrum authorization models, we next discuss 5G spectrum authorization and develop a new local micro licensing model to facilitate the introduction of the new micro-operator concept.

\section{A. Micro-Operators and the Need for Local Micro Licensing}

Micro-operator concept presented in [4]-[6] and [15] is a potential candidate to provide location specific $5 \mathrm{G}$ services to complement current MNO dominant market. The idea is that the micro-operators deploy their own network infrastructure to provide high quality, localized services to the customers. Specifically, the main target is to deploy their own small-cell networks in specific places such as shopping malls, hospitals, stadiums, industry plants, etc. The reason is, as identified in [1], many of the use cases in $5 \mathrm{G}$ will be originated from the above mentioned locations. For example, in the case of a hospital network that facilitates Internet-of-medical things (remote patient monitoring, smart pharmaceuticals, etc.), the required capacity and latency requirements must be highly guaranteed. On the other hand, in other local areas (shopping malls, factories, etc.) which need high capacity broadband connections to facilitate the customers with advanced user experienced like virtual/augmented reality and machine-tomachine communications, the micro-operators could play a major role as the major indoor connectivity provider. Thus, as discussed above a micro-operator has a high potential in 
providing the required services for some specific uses cases $5 \mathrm{G}$ that appear in different verticals, to complement the MNOs. Therefore, by deploying micro-operator networks in such venues they can ensure high quality context related services while complementing MNOs. It is envisioned that a microoperator can act as a neutral host to provide connectivity services to MNOs by serving MNOs' subscribers in specific locations. On the other hand, it is also possible that a microoperator runs a closed network to serve a specific set of customers, e.g., set of machines in a factory environment. With these two modes, one can think that a hybrid model of above two is also a possible way to operate.

As the objective of introducing the micro-operator concept is to encourage new entrants to enter the market, it is expected that there will be a large number of prospective microoperators [4]. Thus, unlike the traditional cellular mobile communication model, the competition for licenses for certain areas can be extremely high, and on the other hand, there may be no competition at all in some other areas. Hence, the existing spectrum authorization models cannot be directly applied while allocating spectrum to micro-operators. The regulator must facilitate guaranteed spectrum access to the micro-operators, as they are supposed to provide high quality $5 \mathrm{G}$ services to the customers. Thus, the regulator has to define a new spectrum authorization model to allocate $5 \mathrm{G}$ spectrum while continuing with the general objectives to ensure fairness and transparency, and promote competition and innovation in the market.

\section{B. Proposed Micro Licensing Elements}

In this subsection, we apply the spectrum authorization framework developed in Section II to expand the recently proposed spectrum authorization model, i.e. micro licensing [5], [15], to support the deployment of micro-operators.

In the proposed micro licensing model the purpose of use is to provide land mobile services by the micro-operator networks. The micro operator can run a closed network (to provide service to a closed group of subscribers) or an open network for MNO customers, or a mix of both.

The eligible licensee element in micro licensing needs to addresses how to expand the current mobile market to encourage new entrants. The regulator must select the eligible licensees so that it does not restrict the candidate microoperators. Furthermore, it is essential to identify whether an eligible licensee is a telecommunication service provider for whom the rights and obligations from the law applies.

The license awarding procedure in the micro-licensing model needs to accommodate potentially a large number of competitors in some specific locations, and very low competition in other locations. Hence, the main challenge here is how to define a fair and efficient procedure (potentially an auction), which is capable of handling a large and a varying number of applicants in different locations, while balancing the trade-off between the efficient spectrum usage and encouraging new entry. Although the regulator can conduct a competitive bidding process through an auction in high demand areas, it may not be efficient in low demand areas. Thus, in such low spectrum demanding areas the regulator can suggest a minimum usage price based on the spectrum utilization (e.g., amount of customers in that area) so that spectrum will not be underutilized. Another design challenge is to encourage the new entrants to the business and how to be fair while allocating the spectrum. Specifically, the MNOs have a large capital with them, and hence spectrum hoarding by MNOs is possible in some cases, especially in high demanding areas. One possibility to prevent this is by introducing spectrum aggregation limits to the applicants. Next, it is mandatory to identify a suitable band and the size of a channel so that it complies with the $5 \mathrm{G}$ standards to deliver localized services. Finally, as in the development of any new spectrum authorization model, it is advisable the regulator to collect opinions from the stakeholders and other relevant bodies to design an efficient license awarding mechanism.

License area definition in the micro licensing model is another critical fact as the micro-operator concept is inherently addressing local areas - particularly specific buildings. The reason is that the license area in here is not equal to the whole country as in exclusive license case or to a census tract as in CBRS. To come up with an appropriate license area, the regulator can think of utilizing the national availability of data (e.g., population and mobile broadband usage densities, geographic and demographic data). Such data helps to understand the high and low demand areas, how to develop interference management tools to efficiently use the spectrum, etc. Furthermore, in the areas where there is a high demand for indoor networks, the regulator may consider the license area is confined to such a building. In addition, carefully defining the terminologies like the service area and the protection area is essential to manage the interference to and from licensees. It is also necessary to think about permitting geographic aggregation across license areas. For example, if the license area is a considered as a building, in a factory premises which has many such buildings, it would be easier for the license holder to consider the entire factory premises as one license region, if the regulator facilitates such aggregation across the license areas. By doing so the licensee can define a single protection area that will cover all his licensing areas.

Interference coordination among the micro-operators (in both co-channel and adjacent channels) as well as managing potential harmful interference the micro-operators generate towards the incumbents (like in CBRS) is imperative. Hence, the regulator has to develop and apply appropriate tools, which are required for efficient interference coordination. For example, defining suitable interference criteria to protect the incumbents and micro-operators is important to make spectrum available. Furthermore, it is also necessary to identify the requirements for introducing the exclusion and protection zones (or default protection contours), and the relevant parameters to define such zones. There can be licensees with multiple micro-operator licenses in the same or adjacent license areas. Hence, by allowing to merge those adjacent license areas into a single license area, it helps the licensee while defining a protection zone because the licensee can have a single protection zone instead of multiple. By allowing the microoperators to negotiate the allowable interference levels among the co-located or adjacent operators under the scrutiny of the regulator, the spectrum usage efficiency can be improved. 
The technical conditions need to be carefully decided by the regulator to define the technical parameters such as interference criteria, the components of BEM, duplexing type, and specifically the parameters related to indoor deployments.

Obligations in the micro licensing model define the operational conditions and boundaries specific to local deployments. The successful operation of the micro-operator concept heavily depends on the accuracy of the information exchanged between the regulator (or any other entity recommended by the regulator) and the micro-operators. Thus, the licensees must report accurate information like deployment parameters, measurements results, etc., to the regulator. Furthermore, the micro-operators must operate according to the operational instructions given by the regulator that can be changed over the time. Such instructions could be the channel allocation information, transmission power levels (to manage interference), interference criteria, etc. As the main objective of the micro-operator concept is to provide location specific services, the regulator could issue new type of coverage obligations that would ensure good indoor coverage.

Transferability of rights in micro licensing is similar to exclusive licensing and CBRS models. The regulator should facilitate transferring or leasing the spectrum access rights to another license holder to improve flexibility and efficiency of spectrum use.

The license duration in micro licensing can be shorter than existing 15-20 year license durations of exclusive licenses in mobile communications to promote flexibility. This decision depends on the investments needed to become a microoperator. With network virtualization and emerging network as a service operational models, the required infrastructure investments are expected to become lower as it will be possible to lease the required infrastructure as a service based on the local demand, and thus, facilitating shorter license duration.

The successful operation of the micro-operator concept heavily relies on the quality of the spectrum available for a micro-operator. We believe that the presented micro licensing model is useful for a regulator to authorize spectrum for enabling the micro-operator concept.

\section{CONCLUSIONS}

In this paper, we have identified the elements of a spectrum authorization model including purpose of use, eligible licensee, license awarding procedure, interference coordination, technical conditions, license area, obligations, transferability of rights, and license duration. By observing the relations of the identified elements, we have developed a framework to analyze existing and develop new spectrum authorization models. By analyzing the state-of-the-art models, we have demonstrated the usability of the developed framework to capture the essence of existing spectrum authorization models. The key importance of the proposed framework is its vast applicability in authorizing new $5 \mathrm{G}$ bands. We have applied this framework to develop a spectrum authorization model for the newly proposed 5G micro-operator concept, where we introduce the micro licensing elements for granting local spectrum access rights to $5 \mathrm{G}$ networks. Furthermore, our comprehensive investigation on the micro licensing supports to establish the micro-operator concept as a candidate solution for delivering localized $5 \mathrm{G}$ services. A regulator may use this framework as a tool for efficiently authorizing spectrum bands among the stakeholders such that it promotes investment and innovation.

\section{REFERENCES}

[1] 5GPPP, "5G empowering vertical industries: Roadmap paper". The 5G Infrastructure Public Private Partnership (5GPPP). 2016.

[2] Ericsson $\mathrm{AB}$, "5G systems enabling the transformation of industry and society," Tech. Rep., Jan. 2017.

[3] J. Zander, "Beyond the ultra-dense barrier: Paradigm shifts on the road beyond 1000x wireless capacity," IEEE Wireless Commun. Mag., vol. 24, no. 3, pp. 96-102, 2017.

[4] M. Matinmikko, M. Latva-aho, and P. Ahokangas, "Micro operators to boost local service delivery in 5G," Wireless Pers. Commun., vol. 95, no. 1, pp. 69-82, May 2017.

[5] M. Matinmikko, A. Roivainen, M. Latva-aho, and K. Hiltunen, "Interference study of micro licensing for $5 \mathrm{G}$ micro operator small cell deployments," in Int. Conf. on Cognitive Radio Oriented Wireless Net. and Commun., to appear in 2017.

[6] P. Ahokangas, M. Matinmikko, S. Yrjölä \& I. Atkova. Disruptive revenue models for future micro operator driven mobile business ecosystem. The 24th Nordic Academy of Management Conference (NFF), Bodo, Norway, 23-25 August 2017.

[7] S. Bhattarai, J. M. J. Park, B. Gao, K. Bian \& W. Lehr. An Overview of Dynamic Spectrum Sharing: Ongoing Initiatives, Challenges, and a Roadmap for Future Research. IEEE Transactions on Cognitive Communications and Networking, vol. 2, 110-128, 2016

[8] Ministry of Transport and Communications Finland, "Invitation for applications for frequency band allocation: Telecom operating licences for the spectrum 703-733 MHz and 758-788 MHz". 2016

[9] Ofcom, "Improving spectrum access for consumers in the $5 \mathrm{GHz}$ band" document on license-exempt". 2016.

[10] FCC, "Amendment of the commissions rules with regard to commercial operations in the 3550-3650 MHz band, Report and Order and Second Further Notice of Proposed Rulemaking," Doc.No. 12-354, Apr. 2015.

[11] FCC, "Amendment of the commissions rules with regard to commercial operations in the $3550-3650 \mathrm{MHz}$ band, Order on Reconsideration and Second Report and Order," Doc.No. 12-354, May 2016.

[12] ECC, "Licensed Shared Access (LSA)". ECC Report 205. Electronic Communications Committee Committee (ECC) of European Conference of Postal and Telecommunications Administrations (CEPT). 2014.

[13] EC, "Authorisation of electronic communications networks and services". Directive 2002/20/EC of the European Parliament and of the Council. 2002.

[14] EC, "Proposal for a Directive of the European Parliament and of the Council establishing the European Electronic Communications Code. 2016.

[15] M. Matinmikko, M. Latva-aho, P. Ahokangas \& V. Seppänen, "On regulations for 5G: Micro licensing for locally operated networks," Telecommunications Policy, to be published

[16] M. Mustonen, M. Matinmikko, O. Holland, \& D. Roberson. Process model for recent spectrum sharing concepts in policy making. Telecommunications Policy, vol. 41, pp. 391-404, 2017.

[17] P. Cramton, P. 2013. Spectrum auction design. Review of Industrial Organization, 42, 161-190.

[18] T. Kuroda \& M. del Pilar Baquero Forero. The effects of spectrum allocation mechanisms on market outcomes: Auctions vs beauty contests. Telecommunications Policy, Vol. 41, no. 5, 2017, pp. 341-354.

[19] E. Avdic, I. Macaluso, N. Marchetti and L. Doyle, "Census Tract License Areas: Disincentive for Sharing the 3.5GHz Band?," 2016 IEEE Global Communications Conference (GLOBECOM), Washington, DC, 2016, pp. 1-7. 